\section{Butterflies of Vidarbha region, Maharashtra State, central India}

\author{
Ashish D. Tiple
}

Department of Zoology, Entomology Division, RTM Nagpur University Campus, Nagpur, Maharashtra, India Forest Entomology Division, Tropical Forest Research Institute, Jabalpur, Madhya Pradesh 482021, India

Email: ashishdtiple@yahoo.co.in

The Indian sub-region hosts about 1,504 species of butterflies (Gaonkar 1996; Smetacek 1992; Kunte 2009; Roy et al. 2010) of which peninsular India hosts 351, and the Western Ghats 334. In central India, the butterfly diversity reported by D'Abreau (1931) totalled 177 species occurring in the erstwhile Central Provinces (now Madhya Pradesh, Chattisgarh \& Vidarbha).

Vidarbha is the eastern region of Maharashtra State covering Nagpur division and Amravati division. It occupies $31.6 \%$ of the total area of Maharashtra. It borders the state of Madhya Pradesh to the north, Chhattisgarh to the east, the Telangana region of Andhra Pradesh to the south and Marathwada and Khandesh regions of Maharashtra to the west. No published checklist of butterfly species of Vidarbha region is known, hence, the present work was initiated.

Habitats: Vidarbha lies on the northern part of

Date of publication (online): 26 January 2011

Date of publication (print): 26 January 2011

ISSN 0974-7907 (online) | 0974-7893 (print)

Editor: Peter Smetacek

Manuscript details:

Ms \# 02397

Received 29 January 2010

Final received 30 July 2010

Finally accepted 24 November 2010

Citation: Tiple, A.D. (2011). Butterflies of Vidarbha region, Maharashtra State, central India. Journal of Threatened Taxa 3(1): 1469-1477.

Copyright: (c) Ashish D. Tiple 2011. Creative Commons Attribution 3.0 Unported License. JoTT allows unrestricted use of this article in any medium for non-profit purposes, reproduction and distribution by providing adequate credit to the authors and the source of publication.

Acknowledgements: Thanks to Prof. A.M. Khurad, Head, Department of Zoology; RTM Nagpur University and Dr. Nitin Kulkarni, Scientist F Tropical Forest Research Institute, Jabalpur for their comments on an earlier draft and kind encouragement. I thank Dr. R.M. Sharma, Central Regional Station, Zoological Survey of India, Jabalpur for literature and critical suggestions from time to time.

OPEN ACCESS | FREE DOWNLOAD the Deccan Plateau. Unlike the Western Ghats, there are no major hilly areas. The Satpura range lies to the north of Vidarbha in Madhya Pradesh. The Melghat area of Amravati District is the southern offshoot of the Satpura range. Wainganga is the largest of all the Vidarbha rivers. Other major rivers that drain the Vidarbha region are the Wardha and Kanhan rivers which are all tributaries of the Godavari River. To the north, five small rivers, namely Khandu, Khapra, Sipna, Gadga and Dolar along with Purna, are the tributaries of the Tapti River.

From the administrative point of view, Vidarbha comprises 11 districts namely, Amravati, Akola, Bhandara, Buldhana, Chandrapur, Gadchiroli, Gondia, Nagpur, Wardha, Washim and Yavatmal.

Forest type: The forests are well distributed over all the agro-climatic zones. The forest types found in the area are classified as sub-tropical hill forests, tropical moist deciduous forests, tropical dry deciduous forests and lush green deciduous forests (Champion \& Seth 1968), which are home to a variety of flora and fauna. All Maharashtra's tiger reserves are located in Vidarbha. They are Melghat Tiger Reserve in Amravati District, Tadoba Andhari Tiger Reserve in Chandrapur District and Pench Tiger Reserve in Nagpur District.

Climatic conditions: Vidarbha has three main seasons: the wet Monsoon and post-Monsoon season from June to October, the cool dry winter from October to March and the hot dry season from April till the onset of rains. The temperature of Vidarbha ranges from a minimum of $12-25^{\circ} \mathrm{C}$ to a maximum of $30-48^{\circ} \mathrm{C}$ with relative humidity varying from $10-15 \%$ to $60-95 \%$. Annual precipitation is $1700 \mathrm{~mm} 90 \%$ of the precipitation falls in four months, i.e. from June to September (Tiple 2009).

History of butterfly surveys in Central India: In central India, the butterfly diversity was reported earlier by Forsayeth (1884), Swinhoe (1886), Betham (1890, 1891), and Witt (1909). Subsequent works include several species from Madhya Pradesh and Chhattisgarh (Evans 1932; Talbot 1939, 1947; Wynter-Blyth 1957). D'Abreau (1931) documented a total of 177 species occurring in the erstwhile central provinces including Pachmari, Pench and Seoni, Nimar, Hoshangabad, Jabalpur, Burahanpur, Raipur, Bastar, Chanda and Nagpur districts (now Madhya 
Pradesh and Vidarbha). In addition to this, D'Abreau (1931) provided a special list of 92 butterfly species from Nagpur city. In the recent past, several workers have studied butterflies from urban, rural and protected areas of Vidarbha.

Pandharipande (1990) in his preliminary studies listed only 61 butterfly species in Nagpur City, representing eight families. A total of 48 species of butterflies were recorded belonging to 35 genera from Lonar Crater Lake, Buldhana District (Palot \& Soniya 2003); 45 butterflies and 54 moths were reported from Pench Tiger Reserve (Maharashtra) by Singh (2004); 65 species belonging to 52 genera representing seven families from Pench Tiger Reserve, Maharashtra (Sharma \& Radhakrishnan 2004); 45 species belonging to 36 genera representing eight families from Melghat Tiger Reserve (Sharma \& Radhakrishnan 2005); 43 species of butterflies of 29 genera from the Tiger Reserve in Tadoba National Park, Maharashtra (Rai et al. 2006); 68 species of butterflies of 50 genera were recorded from Tadoba Andhari Tiger Reserve (Sharma \& Radhakrishnan 2006); 53 species belonging to 36 genera representing seven families from Lonar Wildlife Sanctuary, Buldhana District (Sharma 2008); 53 species of butterflies were recorded from Pohara Malkhed Reserve Forest, Amravati District by Kasambe \& Wadatkar (2004); 52 species of butterflies belonging to five families (22 species to Nymphalidae, 12 to Lycaenidae, 10 to Pieridae, 5 to Papilionidae and 3 species to Hesperiidae) were reported from Amravati University Campus, Maharashtra (Tiple et al. 2006, 2007); 51 butterfly species were recorded belonging to seven families from Melghat Tiger Reserve, Maharashtra (Chandrakar et al. 2007); 101 species of butterflies of eight families and 19 subfamilies were recorded (22 species of Nymphalidae, 6 of Danaidae, 10 of Satyridae, 23 of Lycaenidae, 1 of Riodinidae, 16 of Pieridae, 9 of Papilionidae, and 14 species of Hesperiidae) from Melghat Tiger Reserve (Wadatkar \& Kasambe 2009); 103 species of butterflies belonging to eight families and 19 subfamilies were recorded from Melghat Tiger Reserve (Wadatkar 2008); and 98 species of butterflies belonging to Papilionidae (06 species), Pieridae (14 species), Nymphalidae (39 species), Lycaenidae (24 species) and Hesperiidae (15 species) in reserve forest area, Seminary Hill, Nagpur city (Tiple \& Khurad 2009b).

Recently, Tiple \& Khurad (2009a) reported 145 species of butterflies recorded at eight study sites, of which 62 species were new records for Nagpur City. The highest number of butterflies recorded belonged to the family Nymphalidae (51 species) with 17 new records, followed by Lycaenidae (46 species) with 29 new records, Hesperiidae (22 species) with 14 new records, Pieridae (17 species) with four new records and Papilionidae (9 species).

The compilation of all these studies in Vidarbha region and stray records resulted in the enumeration of 167 species of butterflies belonging to 90 genera representing five families and is given in Table 1. The highest number of butterflies recorded belonged to the Nymphalidae (50 species), followed by Lycaenidae (47 species), Hesperiidae (34 species), Pieridae (23species) and Papilionidae (13 species). All scientific names follow reports by Varshney (1983); Kunte (2000) and common English names are after Wynter-Blyth (1957). Continuous exploration in Vidarbha region could add many more new records for the region.

Among the 167 butterflies recorded from Vidarbha region, 14 species come under the protected category of the Indian Wildlife (Protection) Act, 1972. Among them Pachliopta hector and Hypolimnas misippus come under Schedule I of the Act. The species recorded which come under Schedule II are Hypolimnas misippus, Eurema andersonii, Appias albina, Tanaecia lepidea, Spindasis elima, Melanitis zitenius, Euchrysops cnejus, Ionolyce helicon and Lampides boeticus. The species recorded which come under Schedule IV are Appias libythea, Tarucus ananda, Baoris farri, Euploea core (Kunte 2000; Gupta \& Mondal 2005).

Interestingly, some butterflies (Graphium antiphates, Papilio crino, Ypthima avanta, Everes argiades and Hasora chabrona) which were recorded earlier by D'Abreau (1931) from Vidarbha (Nagpur city) were not seen in recent years. The probable causes of this could be the loss of habitats by ever expanding urbanization along with the broader climatic changes (Tiple et al. 2007). During the last decade, the city has expanded twice in its circumference causing loss of natural habitats of butterflies. Urban development is expected to have a deleterious impact on butterfly populations, if only because the construction of buildings and concrete replaces or reduces the area of natural and semi-natural habitats. The quality of residual habitats may also be adversely affected by 
Table 1. List of butterflies recorded from Vidarbha region

\begin{tabular}{|c|c|c|c|}
\hline Sno & Common name & Scientific name & Distribution \\
\hline & \multicolumn{2}{|l|}{ Papilionidae (13) } & \\
\hline 1 & Tailed Jay & Graphium agamemnon (Linnaeus) & Common throughout Vidarbha \\
\hline 2 & Common Jay & Graphium doson (C.\& R. Felder) & $\begin{array}{l}\text { Nagpur and Amravati (D’Abreau 1931; Tiple \& Khurad 2009a; } \\
\text { Wadatkar \& Kasambe 2009) }\end{array}$ \\
\hline 3 & Spot Swordtail & Graphium nomius (Esper) & Common throughout Vidarbha \\
\hline 4 & Common Bluebottle & Graphium sarpedon (Linnaeus) & $\begin{array}{l}\text { Nagpur and Amravati (D’Abreau 1931; Chandrakar et al. 2007; Tiple } \\
\& \text { Khurad 2009aa; Wadatkar \& Kasambe 2009) }\end{array}$ \\
\hline 5 & Common Rose & Pachliopta aristolochiae (Fabricius) & Common throughout Vidarbha \\
\hline 6 & Crimson Rose & Pachliopta hector (Linnaeus)* & Common throughout Vidarbha \\
\hline 7 & Malabar Rose & Pachliopta pandiyana Moore & Amravati, Melghat (Chandrakar et al. 2007) \\
\hline 8 & Fivebar Swordtail & Graphium antiphates (Cramer) & Nagpur (D’Abreau 1931) \\
\hline 9 & Common Mime & Papilio clytia (Linnaeus) & Nagpur, Pench National Park (Sharma \& Radhakrishan 2004) \\
\hline 10 & Lime & Papilio demoleus Linnaeus & Common throughout Vidarbha \\
\hline 11 & Blue Mormon & Papilio polymnestor Cramer & $\begin{array}{l}\text { Nagpur, Chandrapur Tadoba National Park (Tiple \& Khurad 2009a; } \\
\text { A. Tiple pers. obs.) }\end{array}$ \\
\hline 12 & Common Mormon & Papilio polytes Linnaeus & Common throughout Vidarbha \\
\hline \multirow[t]{2}{*}{13} & $\begin{array}{l}\text { Common Banded } \\
\text { Peacock }\end{array}$ & Papilio crino Fabricius & Nagpur (D’Abreau 1931) \\
\hline & \multicolumn{2}{|l|}{ Pieridae (23) } & \\
\hline 14 & Pioneer & Anaphaeis aurota (Fabricius) & Common throughout Vidarbha \\
\hline 15 & Common Albatross & Appias albina (Boisduval)* & $\begin{array}{l}\text { Nagpur, Amravati (Sharma \& Radhakrishan 2004; Sharma \& } \\
\text { Radhakrishan 2005; Tiple \& Khurad 2009a) }\end{array}$ \\
\hline 16 & $\begin{array}{l}\text { Eastern Striped } \\
\text { Albatross }\end{array}$ & Appias libythea (Fabricius)* & Nagpur (Tiple \& Khurad 2009a) \\
\hline 17 & Lemon Emigrant & Catopsilia pomona (Fabricius) & Common throughout Vidarbha \\
\hline 18 & Mottled Emigrant & Catopsilia pyranthe (Linnaeus) & Common throughout Vidarbha \\
\hline 19 & Common Gull & Cepora nerissa (Fabricius) & Common throughout Vidarbha \\
\hline 20 & Small Salmon Arab & Colotis amata (Butler) & Nagpur, Buldana (D’Abreau 1931, Sharma 2008) \\
\hline 21 & Crimson Tip & Colotis danae (Fabricius) & $\begin{array}{l}\text { Nagpur, Amravati \& Buldana, (D’Abreau 1931; Chandrakar et al. } \\
\text { 2007; Sharma 2008; Tiple \& Khurad 2009a; Wadatkar \& Kasambe } \\
\text { 2009;) }\end{array}$ \\
\hline 22 & Small Orange Tip & Colotis etrida (Boisduval) & $\begin{array}{l}\text { Nagpur, Amravati, Buldana (D’Abreau 1931; Sharma \& Radhakrishan } \\
\text { 2004; Sharma 2008; Tiple \& Khurad 2009a; Wadatkar \& Kasambe } \\
\text { 2009) }\end{array}$ \\
\hline 23 & Plain Orange Tip & Colotis eucharis (Fabricius) & Buldana (Sharma 2008) \\
\hline 24 & Large Salmon Arab & Colotis fausta (Oliver) & Buldana (Sharma 2008) \\
\hline 25 & Common Jezebel & Delias eucharis (Linnaeus) & Common throughout Vidarbha \\
\hline 26 & Painted Jezebel & Delias hyparete (Linnaeus) & Gadchiroli (Bhamaragad forest) Tiple Personal obsevation. \\
\hline 27 & One-spot Grass Yellow & Eurema andersonii (Moore)* & Nagpur (Tiple \& Khurad 2009a) \\
\hline 28 & $\begin{array}{l}\text { Three-spot Grass } \\
\text { Yellow }\end{array}$ & Eurema blanda (Boisduval) & $\begin{array}{l}\text { Nagpur, Amravati, Chandrapur (Sharma \& Radhakrishan 2004, 2006; } \\
\text { Tiple \& Khurad 2009a; Wadatkar \& Kasambe 2009) }\end{array}$ \\
\hline 29 & Small Grass Yellow & Eurema brigitta (Cramer) & Common throughout Vidarbha \\
\hline 30 & Common Grass Yellow & Eurema hecabe (Linnaeus) & Common throughout Vidarbha \\
\hline 31 & Spotless Grass Yellow & Eurema laeta (Boisduval) & Common throughout Vidarbha \\
\hline 32 & Great Orange Tip & Hebomoea glaucippe (Linnaeus) & $\begin{array}{l}\text { Amravati, Melghat (Sharma \& Radhakrishan 2005; Wadatkar \& } \\
\text { Kasambe 2009) }\end{array}$ \\
\hline 33 & White Orange Tip & Ixias marianne (Cramer) & $\begin{array}{l}\text { Nagpur, Amravati, Chandrapur (D’Abreau 1931; Sharma \& } \\
\text { Radhakrishan 2004, 2005, 2006; Tiple et al. 2007; Tiple \& Khurad } \\
\text { 2009a; Wadatkar \& Kasambe 2009) }\end{array}$ \\
\hline 34 & Yellow Orange Tip & Ixias pyrene (Linnaeus) & $\begin{array}{l}\text { Nagpur and Amravati (Sharma \& Radhakrishan 2005, 2006; } \\
\text { Chandrakar et al. 2007; Tiple et al. 2007; Wadatkar \& Kasambe 2009; } \\
\text { Tiple \& Khurad 2009a) }\end{array}$ \\
\hline
\end{tabular}




\begin{tabular}{|c|c|c|c|}
\hline Sno & Common name & Scientific name & Distribution \\
\hline 35 & Psyche & Leptosia nina (Fabricius) & Buldana (Sharma 2008) \\
\hline \multirow[t]{2}{*}{36} & Common Wanderer & Pareronia valeria (Cramer) & $\begin{array}{l}\text { Nagpur, Amravati, Buldana, Chandrapur (Sharma \& Radhakrishan } \\
\text { 2005, 2006; Chandrakar et al. 2007; Tiple et al. 2007; Wadatkar \& } \\
\text { Kasambe 2009; Sharma 2008; Tiple \& Khurad 2009a) }\end{array}$ \\
\hline & \multicolumn{2}{|l|}{ Nymphalidae (50) } & \\
\hline 37 & Plain Tiger & Danaus chrysippus (Linnaeus) & Common throughout Vidarbha \\
\hline 38 & Striped Tiger & Danaus genutia (Cramer) & Common throughout Vidarbha \\
\hline 39 & Glassy Tiger & Parantica aglea (Stoll) & $\begin{array}{l}\text { Nagpur, Amravati (Sharma \& Radhakrishan 2005; Tiple et al. 2006; } \\
\text { Tiple \& Khurad 2009a; Wadatkar \& Kasambe 2009) }\end{array}$ \\
\hline 40 & Blue Tiger & Tirumala limniace (Cramer) & Common throughout the Vidarbha \\
\hline 41 & Dark Blue Tiger & Tirumala septentrionis (Butler) & $\begin{array}{l}\text { Nagpur, Amravati, Buldana (D'Abreau 1931; Sharma 2008; Tiple \& } \\
\text { Khurad 2009a; Wadatkar \& Kasambe 2009) }\end{array}$ \\
\hline 42 & Common Indian Crow & Euploea core (Cramer)* & Common throughout Vidarbha \\
\hline 43 & Brown King Crow & Euploea klugii Moore & Nagpur (Tiple \& Khurad 2009a) \\
\hline 44 & Bamboo Treebrown & Lethe europa (Fabricius) & $\begin{array}{l}\text { Nagpur, Amravati (D'Abreau 1931; Tiple \& Khurad 2009a; Wadatkar \& } \\
\text { Kasambe 2009) }\end{array}$ \\
\hline 45 & Common Treebrown & Lethe rohria (Fabricius) & $\begin{array}{l}\text { Nagpur, Amravati (D’Abreau 1931; Tiple \& Khurad 2009a; Wadatkar \& } \\
\text { Kasambe 2009) }\end{array}$ \\
\hline 46 & $\begin{array}{l}\text { Dark Branded } \\
\text { Bushbrown }\end{array}$ & Mycalesis mineus (Linnaeus) & Common throughout Vidarbha \\
\hline 47 & Common Bushbrown & Mycalesis perseus (Fabricius) & Common throughout Vidarbha \\
\hline 48 & Tamil Bushbrown & Mycalesis subdita (Moore) & Nagpur, Amravati (Tiple \& Khurad 2009a; Wadatkar \& Kasambe 2009) \\
\hline 49 & Longbrand Bushbrown & Mycalesis visala Moore & Nagpur (Tiple \& Khurad 2009a) \\
\hline 50 & Nigger & Orsotriaena medus Fabricius & Nagpur (D’Abreau 1931; Tiple \& Khurad 2009a) \\
\hline 51 & $\begin{array}{l}\text { Common Evening } \\
\text { Brown }\end{array}$ & Melanitis leda (Linnaeus) & Common throughout Vidarbha \\
\hline 52 & Dark Evening Brown & Melanitis phedima (Cramer) & Nagpur, Amravati (Tiple \& Khurad 2009a; Wadatkar \& Kasambe 2009) \\
\hline 53 & Great Evening Brown & Melanitis zitenius ( Herbst)* & Nagpur (Tiple \& Khurad 2009a) \\
\hline 54 & Common Threering & Ypthima asterope (Klug) & $\begin{array}{l}\text { Nagpur, Amravati, Buldana, Chandrapur (D’Abreau 1931; Sharma } \\
\text { \& Radhakrishan 2004, 2006; Tiple \& Khurad 2009a; Wadatkar \& } \\
\text { Kasambe 2009) }\end{array}$ \\
\hline 55 & Lesser Threering & Ypthima inica (Hewitson) & Nagpur (Tiple \& Khurad 2009a) \\
\hline 56 & Common Four Ring & Ypthima huebneri Kirby & $\begin{array}{l}\text { Nagpur, Amravati, Bhandara, Chandrapur (D’Abreau 1931; Sharma } \\
\text { \& Radhakrishan 2004, 2005, 2006; Sharma 2008; Tiple \& Khurad } \\
\text { 2009a; Wadatkar \& Kasambe 2009) }\end{array}$ \\
\hline 57 & Jewel Fourring & Ypthima avanta Moore & Nagpur (D’Abreau 1931) \\
\hline 58 & Common Fivering & Ypthima baldus (Fabricius) & $\begin{array}{l}\text { Nagpur, Chandrapur (Tiple \& Khurad 2009a; Sharma \& Radhakrishan } \\
2006 \text { ) }\end{array}$ \\
\hline 59 & Tawny Rajah & Charaxes psaphon Westwood & $\begin{array}{l}\text { Nagpur and Amravati (D’Abreau 1931; Tiple \& Khurad 2009a; } \\
\text { Wadatkar \& Kasambe 2009) }\end{array}$ \\
\hline 60 & Black Rajah & Charaxes solon (Fabricius) & $\begin{array}{l}\text { Nagpur, Amravati, Chandrapur (D’Abreau 1931; Sharma \& } \\
\text { Radhakrishan 2006; Tiple \& Khurad 2009a; Wadatkar \& Kasambe } \\
\text { 2009) }\end{array}$ \\
\hline 61 & Common Nawab & Polyura athamas (Drury) & $\begin{array}{l}\text { Nagpur, Amravati, Chandrapur, Buldana (D’Abreau 1931; Sharma \& } \\
\text { Radhakrishan 2006; Sharma 2008; Tiple \& Khurad 2009a; Wadatkar } \\
\text { \& Kasambe 2009) }\end{array}$ \\
\hline 62 & Anomalous Nawab & Polyura agraria Swinhoe & Nagpur (Tiple \& Khurad 2009a) \\
\hline 63 & Common Baron & Euthalia aconthea (Cramer) & $\begin{array}{l}\text { Nagpur, Amravati and Buldana, Chandrapur (Sharma \& Radhakrishan } \\
\text { 2005, 2006; Tiple et al. 2007; Chandrakar et al. 2007; Sharma 2008; } \\
\text { Tiple \& Khurad 2009a; Wadatkar \& Kasambe 2009) }\end{array}$ \\
\hline 64 & Gaudy Baron & Euthalia lubentina (Cramer) & Nagpur, Pench National Park (Sharma \& Radhakrishan 2004) \\
\hline 65 & Baronet & Symphaedra nais (Forster) & $\begin{array}{l}\text { Nagpur, Amravati, Buldana, Chandrapur (Sharma \& Radhakrishan } \\
\text { 2004, 2005, 2006; Tiple et al. 2007; Sharma 2008; Tiple \& Khurad } \\
\text { 2009a; Wadatkar \& Kasambe 2009) }\end{array}$ \\
\hline 66 & Grey Count & Tanaecia lepidea (Butler)* & Bhamaragad forest in Gadchiroli (Published in local Newspaper) \\
\hline 67 & Great Eggfly & Hypolimnas bolina (Linnaeus) & Common throughout Vidarbha \\
\hline
\end{tabular}




\begin{tabular}{|c|c|c|c|}
\hline Sno & Common name & Scientific name & Distribution \\
\hline 68 & Danaid Eggfly & Hypolimnas misippus (Linnaeus)* & Common throughout Vidarbha \\
\hline 69 & Commander & Moduza procris (Cramer) & $\begin{array}{l}\text { Nagpur, Amravati, Buldana, Chandrapur (D'Abreau 1931; Sharma \& } \\
\text { Radhakrishan 2004, 2005, 2006; Chandrakar et al. 2007; Tiple et al. } \\
\text { 2007; Tiple \& Khurad 2009a; Wadatkar \& Kasambe 2009) }\end{array}$ \\
\hline 70 & Common Sergeant & Athyma perius (Linnaeus) & $\begin{array}{l}\text { Nagpur, Amravati (D’Abreau 1931; Tiple \& Khurad 2009a; Wadatkar \& } \\
\text { Kasambe 2009) }\end{array}$ \\
\hline 71 & Staff Sergeant & Athyma selenophora (Kollar) & Amravati (Wadatkar \& Kasambe 2009) \\
\hline 72 & Short Banded Sailer & Neptis columella (Cramer) & Nagpur (D’Abreau 1931; Tiple \& Khurad 2009a) \\
\hline 73 & Common Sailer & Neptis hylas (Linnaeus) & Common throughout the Vidarbha \\
\hline 74 & $\begin{array}{l}\text { Chestnut-Streaked } \\
\text { Sailer }\end{array}$ & Neptis jumbah Moore & Nagpur (Tiple \& Khurad 2009a) \\
\hline 75 & Painted Lady & Cynthia cardui (Linnaeus) & Common throughout Vidarbha \\
\hline 76 & Peacock Pansy & Junonia almana (Linnaeus) & Common throughout Vidarbha \\
\hline 77 & Grey Pansy & Junonia atlites (Linnaeus) & Common throughout Vidarbha \\
\hline 78 & Yellow Pansy & Junonia hierta (Fabricius) & Common throughout Vidarbha \\
\hline 79 & Chocolate Pansy & Junonia iphita (Cramer) & Common throughout Vidarbha \\
\hline 80 & Lemon Pansy & Junonia lemonias (Linnaeus) & Common throughout Vidarbha \\
\hline 81 & Blue Pansy & Junonia orithya (Linnaeus) & Common throughout Vidarbha \\
\hline 82 & Common Leopard & Phalanta phalantha (Drury) & Common throughout Vidarbha \\
\hline 83 & Angled Castor & Ariadne ariadne (Linnaeus) & Common throughout Vidarbha \\
\hline 84 & Common Castor & Ariadne merione (Cramer) & Common throughout Vidarbha \\
\hline 85 & Joker & Byblia ilithyia (Drury) & $\begin{array}{l}\text { Nagpur, Amravati, Chandrapur (Sharma \& Radhakrishan 2006; Tiple } \\
\text { et al. 2007; Tiple \& Khurad 2009a; Wadatkar \& Kasambe 2009) }\end{array}$ \\
\hline \multirow[t]{2}{*}{86} & Tawny Coster & Acraea violae (Fabricius) & Common throughout Vidarbha \\
\hline & \multicolumn{2}{|l|}{ Lycaenidae (48) } & \\
\hline 87 & Plum Judy & Abisara echerius (Stoll) & $\begin{array}{l}\text { Nagpur, Amravati (D'Abreau 1931; Sharma \& Radhakrishan 2005; } \\
\text { Tiple \& Khurad 2009a; Wadatkar \& Kasambe 2009) }\end{array}$ \\
\hline 88 & Red Pierrot & $\begin{array}{l}\text { Talicada nyseus (Guérin- } \\
\text { Menéville) }\end{array}$ & Nagpur (D’Abreau 1931; Tiple \& Khurad 2009a) \\
\hline 89 & Common Pierrot & Castalius rosimon (Fabricius) & Common throughout Vidarbha \\
\hline 90 & Dark Pierrot & Tarucus ananda de Nicéville * & Nagpur, Amravati (Tiple \& Khurad 2009a; Wadatkar \& Kasambe 2009) \\
\hline 91 & Rounded Pierrot & Tarucus nara Kollar & Common throughout Vidarbha \\
\hline 92 & Zebra Blue & Leptotes plinius Fabricius & Common throughout Vidarbha \\
\hline 93 & African Babul Blue & Azanus jesous (Guérin- Menéville) & $\begin{array}{l}\text { Nagpur, Amravati (D’Abreau 1931; Tiple \& Khurad 2009a; Wadatkar \& } \\
\text { Kasambe 2009) }\end{array}$ \\
\hline 94 & Bright Babul Blue & Azanus ubaldus (Stoll) & Nagpur, Buldana (Sharma 2008; Tiple \& Khurad 2009a) \\
\hline 95 & Dull Babul Blue & Azanus uranus Butler & Nagpur (D’Abreau 1931; Tiple \& Khurad 2009a) \\
\hline 96 & Indian Cupid & Everes lacturnus (Godart) & Nagpur (Tiple \& Khurad 2009a) \\
\hline 97 & Common Hedge Blue & Acytolepis puspa (Horsfield) & $\begin{array}{l}\text { Nagpur, Amravati (D'Abreau 1931; Tiple \& Khurad 2009a; Wadatkar \& } \\
\text { Kasambe 2009) }\end{array}$ \\
\hline 98 & Plain Hedge Blue & Celastrina lavendularis (Moore) & $\begin{array}{l}\text { Nagpur, Chandrapur (Sharma \& Radhakrishan 2004, 2006; Tiple \& } \\
\text { Khurad 2009a) }\end{array}$ \\
\hline 99 & Lime Blue & Chilades laius (Stoll) & Common throughout Vidarbha \\
\hline 100 & Plains Cupid & Chilades pandava (Horsfield) & Common throughout Vidarbha \\
\hline 101 & Small Cupid & Chilades parrhasius (Butler) & $\begin{array}{l}\text { Nagpur, Amravati (D’Abreau 1931; Tiple \& Khurad 2009a; Wadatkar \& } \\
\text { Kasambe 2009) }\end{array}$ \\
\hline 102 & Eastern Grass Jewel & Chilades putli Kollar & Nagpur (Tiple \& Khurad 2009a) \\
\hline 103 & Grass Jewel & Chilades trochylus Freyer & Common throughout Vidarbha \\
\hline 104 & Dark Grass Blue & Zizeeria karsandra (Moore) & Common throughout Vidarbha \\
\hline 105 & Lesser Grass Blue & Zizina otis (Fabricius) & Common throughout Vidarbha \\
\hline
\end{tabular}




\begin{tabular}{|c|c|c|c|}
\hline Sno & Common name & Scientific name & Distribution \\
\hline 106 & Pale Grass Blue & Psuedozizeeria maha (Kollar) & $\begin{array}{l}\text { Nagpur, Amravati, Chandrapur, Buldana (D'Abreau 1931; Sharma } \\
\text { \& Radhakrishan 2004, 2006; Sharma 2008; Tiple \& Khurad 2009a; } \\
\text { Wadatkar \& Kasambe 2009) }\end{array}$ \\
\hline 107 & Tiny Grass Blue & Zizula hylax (Fabricius) & Common throughout Vidarbha \\
\hline 108 & Gram Blue & Euchrysops cnejus (Fabricius)* & Common throughout Vidarbha \\
\hline 109 & Ciliate Blue & Anthene emolus (Godart) & Nagpur (Tiple \& Khurad 2009a) \\
\hline 110 & Pointed Ciliate Blue & Anthene lycaenina (C.\&R. Felder) & Nagpur (Tiple \& Khurad 2009a) \\
\hline 111 & Forget-me-Not & Catochrysops strabo (Fabricius) & Common throughout Vidarbha \\
\hline 112 & Pea Blue & Lampides boeticus (Linnaeus)* & $\begin{array}{l}\text { Common throughout Vidarbha (D'Abreau } 1931 \text { as Polyommatus } \\
\text { boeticus) }\end{array}$ \\
\hline 113 & Cerulean & Jamides alecto (C.\&R. Felder)* & Nagpur (Tiple \& Khurad 2009a) \\
\hline 114 & Dark Cerulean & Jamides bochus (Stoll) & $\begin{array}{l}\text { Nagpur, Chandrapur (Sharma \& Radhakrishan 2004, 2006; Tiple \& } \\
\text { Khurad 2009a) }\end{array}$ \\
\hline 115 & Common Cerulean & Jamides celeno (Cramer) & Common throughout Vidarbha \\
\hline 116 & Pointed Line Blue & Ionolyce helicon (C.\&R. Felder)* & Nagpur (Tiple \& Khurad 2009a) \\
\hline 117 & Opaque 6-Line Blue & Nacaduba beroe (C.\&R. felder) & Nagpur (Tiple \& Khurad 2009a) \\
\hline 118 & Transparent 6-Line Blue & Nacaduba kurava (Moore) & Nagpur (Tiple \& Khurad 2009a) \\
\hline 119 & Dingy Line Blue & Petrelaea dana (de Nicéville) & Nagpur (Tiple \& Khurad 2009a) \\
\hline 120 & Tailless Line Blue & Prosotas dubiosa Evans & Nagpur (Tiple \& Khurad 2009a) \\
\hline 121 & Common Line Blue & Prosotas nora (C. Felder) & Common throughout the Vidarbha \\
\hline 122 & Leaf Blue & Amblypodia anita (Hewitson) & Nagpur (Tiple \& Khurad 2009a) \\
\hline 123 & Large Oakblue & Arhopala amantes (Hewitson) & $\begin{array}{l}\text { Nagpur, Amravati, Chandrapur (D’Abreau 1931; Sharma \& } \\
\text { Radhakrishan 2006; Tiple \& Khurad 2009a; Wadatkar \& Kasambe } \\
\text { 2009) }\end{array}$ \\
\hline 124 & $\begin{array}{l}\text { Western Centaur } \\
\text { Oakblue }\end{array}$ & $\begin{array}{l}\text { Arhopala pseudocentaurus } \\
\text { (Doubleday) }\end{array}$ & Amravati (Wadatkar \& Kasambe 2009) \\
\hline 125 & Common Acacia Blue & Surendra quercetorum (Moore) & Chandrapur (Sharma \& Radhakrishan 2006) \\
\hline 126 & Scarce Shot Silverline & Spindasis elima Moore* & Nagpur (Tiple \& Khurad 2009a) \\
\hline 127 & Shot Silverline & Spindasis ictis Hewitson & Nagpur, Buldana (Sharma 2008; Tiple \& Khurad 2009a) \\
\hline 128 & Plumbeous Silverline & Spindasis schistacea (Moore) & Nagpur (Tiple \& Khurad 2009a) \\
\hline 129 & Common Silverline & Spindasis vulcanus (Fabricius) & $\begin{array}{l}\text { Nagpur, Amravati (D’Abreau 1931; Sharma \& Radhakrishan 2004; } \\
\text { Tiple \& Khurad 2009a; Wadatkar \& Kasambe 2009) }\end{array}$ \\
\hline 130 & Common Guava Blue & Virachola isocrates (Fabricius) & Nagpur (Tiple \& Khurad 2009a) \\
\hline 131 & Indian Red Flash & Rapala iarbus (Fabricius) & $\begin{array}{l}\text { Nagpur, Amravati (D’Abreau 1931; Tiple et al. 2006; Tiple \& Khurad } \\
\text { 2009a; Wadatkar \& Kasambe 2009) }\end{array}$ \\
\hline \multirow[t]{2}{*}{132} & Slate Flash & Rapala manea (Hewitson) & $\begin{array}{l}\text { Nagpur, Amravati (D’Abreau 1931; Tiple et al. 2006; Tiple \& Khurad } \\
\text { 2009a; Wadatkar \& Kasambe 2009) }\end{array}$ \\
\hline & \multicolumn{2}{|l|}{ Hesperiidae (34) } & \\
\hline 133 & Common Banded Awl & Hasora chromus (Cramer) & $\begin{array}{l}\text { Nagpur, Chandrapur, Amravati (Sharma \& Radhakrishan 2004, 2006; } \\
\text { Tiple \& Khurad 2009a; Wadatkar \& Kasambe 2009) }\end{array}$ \\
\hline 134 & Common Awl & Hasora badra (Moore) & Amravati (Sharma \& Radhakrishan 2005) \\
\hline 135 & White Banded Awl & Hasora taminatus Hubner & Nagpur (D’Abreau 1931; Tiple \& Khurad 2009a) \\
\hline 136 & Plain Banded Awl & Hasora vitta (Butler) & Nagpur (D’Abreau 1931) \\
\hline 137 & Brown Awl & Badamia exclamationis (Fabricius) & $\begin{array}{l}\text { Nagpur, Amravati, Chandrapur (A. Tiple pers. obs.; Sharma \& } \\
\text { Radhakrishan 2004, } 2006 \text {; Tiple et al. 2007; Wadatkar \& Kasambe } \\
\text { 2009) }\end{array}$ \\
\hline 138 & Common Spotted Flat & Celaenorrhinus leucocera (Kollar) & Nagpur, Amravati (D’Abreau 1931; Wadatkar \& Kasambe 2009) \\
\hline 139 & Multi-spotted Flat & Celaenorrhinus pulomaya (Moore) & Pench National Park (A. Tiple pers. obs.) \\
\hline 140 & Fulvous Pied Flat & Pseudocoladenia dan (Fabricius) & Nagpur (Tiple \& Khurad 2009a) \\
\hline 141 & Tricolour Pied Flat & Coladenia indrani (Moore) & Nagpur (Sharma \& Radhakrishan 2004) \\
\hline 142 & Spotted Small Flat & Sarangesa purendra Moore & Amravati (Wadatkar \& Kasambe 2009) \\
\hline 143 & Golden Angle & Caprona ransonnetti (C.\&R. Felder) & Nagpur (Tiple \& Khurad 2009a) \\
\hline 144 & Indian Skipper & Spialia galba (Fabricius) & Common throughout Vidarbha \\
\hline
\end{tabular}




\begin{tabular}{|c|c|c|c|}
\hline Sno & Common name & Scientific name & Distribution \\
\hline 145 & Bush Hopper & Ampittia dioscorides (Fabricius) & Nagpur, Amravati (D’Abreau 1931; Wadatkar \& Kasambe 2009) \\
\hline 146 & Grass Demon & Udaspes folus (Cramer) & $\begin{array}{l}\text { Nagpur, Amravati, Chandrapur (Sharma \& Radhakrishan 2004; Tiple } \\
\text { \& Khurad 2009a; Wadatkar \& Kasambe 2009) }\end{array}$ \\
\hline 147 & Vindhyan Bob & Arnetta vindhiana (Moore) & Amravati (Wadatkar \& Kasambe 2009) \\
\hline 148 & Chestnut Bob & lambrix salsala (Moore) & Nagpur, Amravati (D’Abreau 1931; Wadatkar \& Kasambe 2009) \\
\hline 149 & Indian Palm Bob & Suastus gremius (Fabricius) & $\begin{array}{l}\text { Nagpur, Amravati, Chandrapur, Buldana (D’Abreau 1931; Sharma \& } \\
\text { Radhakrishan 2006; Tiple et al. 2007; Sharma 2008; Tiple \& Khurad } \\
\text { 2009a; Wadatkar \& Kasambe 2009) }\end{array}$ \\
\hline 150 & Common Redeye & Matapa aria (Moore) & Chandrapur (Sharma \& Radhakrishan 2006) \\
\hline 151 & Tamil Grass Dart & Taractrocera ceramas (Hewitson) & Nagpur (Tiple \& Khurad 2009a) \\
\hline 152 & Common Grass Dart & Taractrocera maevius (Fabricius) & Nagpur (D’Abreau 1931; Tiple \& Khurad 2009a) \\
\hline 153 & Indian/Common Dartlet & Oriens goloides (Moore) & $\begin{array}{l}\text { Nagpur, Amravati, Chandrapur (D’Abreau 1931; Sharma \& } \\
\text { Radhakrishan 2006; Tiple \& Khurad 2009a; Wadatkar \& Kasambe } \\
\text { 2009) }\end{array}$ \\
\hline 154 & Common Dart & Potanthus pseudomaesa & Nagpur Ramtek (D’Abreau 1931; A. Tiple pers. obs.) \\
\hline 155 & Dark Palm Dart & Telicota ancilla (Herrich-Schäffer) & $\begin{array}{l}\text { Nagpur, Chandrapur (Sharma \& Radhakrishan 2004, 2006; Tiple \& } \\
\text { Khurad 2009a) }\end{array}$ \\
\hline 156 & Pale Palm Dart & Telicota colon (Fabricius) & Nagpur (D’Abreau 1931; Tiple \& Khurad 2009a) \\
\hline 157 & Paintbrush Swift & Baoris farri (Moore)* & Nagpur (D’Abreau 1931; Tiple \& Khurad 2009a) \\
\hline 158 & Bevan's Swift & Borbo bevani (Moore) & Nagpur (D’Abreau 1931; Tiple \& Khurad 2009a) \\
\hline 159 & Rice Swift & Borbo cinnara (Wallace) & Common throughout Vidarbha \\
\hline 160 & Conjoined Swift & $\begin{array}{l}\text { Pelopidas conjuncta (Herrich- } \\
\text { Schaffer) }\end{array}$ & Nagpur (Tiple \& Khurad 2009a) \\
\hline 161 & Small Branded Swift & Pelopidas mathias (Fabricius) & $\begin{array}{l}\text { Nagpur, Amravati (D'Abreau 1931; Tiple \& Khurad 2009a; Wadatkar \& } \\
\text { Kasambe 2009) }\end{array}$ \\
\hline 162 & Large Branded Swift & Pelopidas subochracea (Moore) & $\begin{array}{l}\text { Nagpur, Chandrapur (Sharma \& Radhakrishan 2004, 2006; Tiple \& } \\
\text { Khurad 2009a) }\end{array}$ \\
\hline 163 & Contiguous Swift & $\begin{array}{l}\text { Polytremis lubricans (Herrich- } \\
\text { Schäffer) }\end{array}$ & Nagpur (Tiple \& Khurad 2009a) \\
\hline 164 & Straight Swift & Parnara naso (Bremer \& Grey) & Nagpur (Tiple \& Khurad 2009a) \\
\hline 165 & Kanara Swift & Caltoris canaraica (Moore) & Nagpur (Tiple \& Khurad 2009a) \\
\hline 166 & Blank Swift & Caltoris kumara (Moore) & $\begin{array}{l}\text { Nagpur, Chandrapur (Sharma \& Radhakrishan 2006; Tiple \& Khurad } \\
\text { 2009a) }\end{array}$ \\
\hline
\end{tabular}

* protected under Indian Wild Life (Protection) Act 1972

Table 2. Unusual records for the Vidarbha region

\begin{tabular}{|c|c|c|}
\hline Common Name & Scientific Name & Distributions \\
\hline \multicolumn{3}{|l|}{ Papilionidae } \\
\hline Spangle & Papilio protenor Cramer & Amravati, Melghat (Chandrakar et al. 2007) \\
\hline \multicolumn{3}{|l|}{ Pieridae } \\
\hline Indian Cabbage White & Pieris canidia (Sparrman) & Nagpur, Pench National Park (Sharma \& Radhakrishan 2004) \\
\hline \multicolumn{3}{|l|}{ Nymphalidae } \\
\hline Yellow Rajah & Charaxes marmax Westwood & Nagpur City (Tiple \& Khurad 2009a) \\
\hline Banded Treebrown & Lethe confusa Aurivillius & Amravati Melghat (Chandrakar et al. 2007; Wadatkar \& Kasambe 2009) \\
\hline Whiteline Bushbrown & Mycalesis malsara (Moore) & $\begin{array}{l}\text { Nagpur City, Amravati Melghat (D’Abreau 1931; Tiple \& Khurad 2009a; Wadatkar \& } \\
\text { Kasambe 2009) }\end{array}$ \\
\hline \multicolumn{3}{|l|}{ Lycaenidae } \\
\hline Tailed Cupid & Everes argiades (Pallas) & $\begin{array}{l}\text { Nagpur (D'Abreau 1931). This is probably misidentified, since E. argiades is a } \\
\text { Himalayan taxon. It was probably E. lacturnus that D'Abreau recorded. }\end{array}$ \\
\hline
\end{tabular}


various forms of pollutants (Dennis \& Williams 1986; Tiple \& Khurad 2009b).

Some authors have reported rather unusual records for the region. These reports were checked and found to be based on sightings and field identifications. Since it appeared that these reports could possibly be based on misidentified butterflies, it was thought better to include these species in a separate table (Table 2) pending confirmation of their presence in the study area. Table 2 therefore contains unsubstantiated new records for the region which have been reported in the literature.

\section{REFERENCES}

Betham, J.A. (1890). The butterflies of the Central Provinces. Journal of the Bombay Natural History Society 5: 19-28; 151-161; 279-286.

Betham, J.A. (1891). The butterflies of the Central Provinces. Journal of the Bombay Natural History Society 6: 175-183; 318-331.

Champion, H.G. \& S.K. Seth (1968). A Revised Survey of The Forest Types of India. Government of India Printing Press, New Delhi, 404pp.

Chandrakar, M., S. Palekar \& S. Chandrakar (2007). Butterfly fauna of Melghat Region, Maharashtra. Zoos' Print Journal 22(7): 2762-2764.

D'Abreau, E.A. (1931). The Central Provinces Butterfly List. Records of the Nagpur Museum Number VII. Government Printing Press, Nagpur City, 1-39pp.

Dennis, R.L.H. \& W.R. Williams (1986). Mate locating behaviour of the large skipper butterfly Ochlodes venata: flexible strategies and spatial components. Journal of the Lepidopterists' Society 41: 45-64.

Evans, W.H. (1932). The Identification of Indian butterflies. 2nd ed. Bombay Natural History Society, Bombay, $\mathrm{x}+454 \mathrm{pp}+32 \mathrm{pls}$.

Forsayeth, R.W. (1884). Life history of sixty species of Lepidoptera observed in Mhow, Central India. Transactions of the Entomological Society of London 3: 377-419.

Gaonkar, H. (1996). Butterflies of Western Ghats with notes on those of SriLanka. A report to the Center of Ecological Sciences, Indian Institute of Science, Bangalore, Zoological Museum, Copenhagen and Natural History Museum, London, 89pp.

Gupta, I.J. \& D.K. Mondal (2005). Red Data Book, Part II: Butterflies of India. Zoological Survey of India, Kolkata, $\mathrm{xv}+535 \mathrm{pp}$.

Kasambe, R. \& J. S. Wadatkar (2004). Butterflies of Pohara Malkhed Reserve Forest, Amravati District (Maharashtra). Bugs ' $R$ ' All 7(2): 5-6.

Kunte, K. ( 2000). Butterflies of Peninsular India. Universities
Press (Hyderabad) and Indian Academy of Sciences (Bangalore), xviii+254pp.

Kunte K. (2009). Occurrence of Elymnias obnubila Marshall and de Nicéville, 1883 (Lepidoptera: Nymphalidae: Satyrinae) in southern Mizoram: Range extension of the species and an addition to the Indian butterfly fauna. Journal of Threatened Taxa 1(11): 567-568.

Palot, M.J. \& V.P. Soniya (2003). A preliminary report on the Butterflies of Lonar Crater Lake, Buldhana District, Maharashtra. Zoos' Print Journal 18(11): 1267-1268.

Pandharipande, T.N. (1990). Butterflies from Nagpur City, Central India (Lepidoptera: Rhopalocera). Journal of Research on the Lepidoptera 29(1/2): 157-160.

Rai, M.M., P. Giradkar, M.K. Rathod \& A.M. Khurad (2006). Biodiversity: Colour pattern and butterfly diversity in Tiger Reserve in Tadoba National Park, Maharashtra, pp. 65-74. In: William, S.J. (ed.). Life to Our Mother Earth. G.M. Offset Press, Chennai.

Roy, A.B., U. Ghosh \& K. Kunte (2010). Sighting of Elymnias panthera (Lepidoptera: Nymphalidae : Satyrinae) in West Bengal, eastern India. Journal of Threatened Taxa 2(1): 670-671.

Sharma, R.M. (2008). Insecta: Lepidoptera (Rhopalocera and Grypocera). Fauna of Lonar Wildlife Sanctuary, Zoological Survey of India. Conservation Area Series 37: 181-190.

Sharma, R.M. \& C. Radhakrishnan (2004). Insecta: Lepidoptera (Rhopalocera and Grypocera). Fauna of Melghat Tiger Reserve. Zoological Survey of India. Conservation Area Series 24: 377-400.

Sharma, R.M. \& C. Radhakrishnan (2005). Insecta: Lepidoptera (Rhopalocera and Grypocera). Fauna of Pench National Park. Zoological Survey of India. Conservation Area Series 20: 251-274.

Sharma, R.M. \& C. Radhakrishnan (2006). Insecta: Lepidoptera (Rhopalocera and Grypocera). Fauna of Tadoba Andhari Tiger Reserve. Zoological Survey of India. Conservation Area Series 25: 255-277.

Singh, J. (2004). Status of tiger conservation in the Pench National Park and Tiger Reserve, Maharashtra. Proceedings of the Symposium on Three Decades of Project Tiger in Melghat 119 -133.

Smetacek, P. (1992). Record of Plebejus eversmanni (Stgr.) from India. Journal of the Bombay Natural History Society 89: 385-386

Swinhoe, C. (1886). On the Lepidoptera of Mhow. Proceedings of the Zoological Society of London, pp.421-465.

Talbot, G. (1939). The Fauna of British India including Ceylon and Burma. Butterflies. Today and Tomorrow's Printers and Publishers, New Delhi, 600pp.

Talbot, G. (1947). The Fauna of British India including Ceylon and Burma. Butterflies. Today and Tomorrow's Printers and Publishers, New Delhi, 506pp.

Tiple, A.D. (2009). Butterflies from Nagpur city, Central India: Diversity, population, nectar and larval host plants and the implications for conservation. Ph.D. Thesis submitted to RTM Nagpur University. pp.145. 
Tiple, A.D. \& A.M. Khurad (2009a). Butterfly Species Diversity, Habitats and Seasonal Distribution in and around Nagpur City, Central India. World Journal of Zoology. 4(3): 153-162.

Tiple, A.D. \& A.M. Khurad (2009b). Butterfly diversity of Seminary Hill, Nagpur (Central India) with their habitat and occurrence. Hislopia 1: 39-44.

Tiple, A.D., A.M. Khurad \& R.L.H. Dennis (2007). Butterfly diversity in relation to a human-impact gradient on an Indian university campus. Nota Lepidopteralogica 30(1): 179-188.

Tiple, A.D., V.P. Deshmukh \& R.L.H. Dennis (2006). Factors influencing nectar plant resource visits by butterflies on a university campus: implications for conservation. Nota Lepidopteralogica 28: 213-224.
Varshney, R.K. (1983). Index Rhopalocera Indica. Part II. Common names of Butterflies from India and neighbouring countries. Records of the Zoological Survey of India. Occasional Paper 47: 1-49.

Wadatkar, J.S. (2008). Butterflies of Melghat Tiger Reserve, Maharashtra with notes on their abundance, status. MelVyaghra XIII(III): 1-5.

Wadatkar, J.S. \& R. Kasambe (2009). Butterflies of Melghat Tiger Reserve, Maharashtra with notes on their abundance, status and larval host plants. The Ecoscan (2):165-171

Witt, D.O. (1909). The butterflies (Rhopalocera) of the Nimar district, Central Provinces. Journal of the Bombay Natural History Society 19(3): 564-571.

Wynter-Blyth, M.A. (1957). Butterflies of the Indian Region. Bombay Natural History Society, 523pp. 